\title{
Current Perspectives: Therapeutic Uses of Tobramycin
}

\author{
Milagros P Reyes ${ }^{1 *}$, Jing J Zhao ${ }^{2}$ and Joseph AL Buensalido'
}

${ }^{1}$ Division of Infectious Diseases, Wayne State University School of Medicine, USA

${ }^{2}$ Department of Pharmacy, Harper University Hospital,Detroit Medical Center, Detroit, Michigan, USA

\begin{abstract}
Tobramycin has been around for over 40 years. This aminoglycoside antibiotic continues to be an invaluable part of our antibiotic armamentarium. It demonstrates concentration-dependent bactericidal activity, post antibiotic effect, and is minimally influenced by bacterial inoculum.
\end{abstract}

\section{Introduction}

Tobramycin was initially identified as part of a broad-spectrum antibiotic complex, nebramycin, which was first isolated in 1967 by Eli Lilly and Company [1]. One component of this complex, first designated as Factor 6, subsequently became tobramycin, since it was derived from Streptomyces tenebrarius [1]. Tobramycin is most similar in structure to members of the kanamycin group of aminoglycosides. Tobramycin inhibits protein synthesis through binding with the $30 \mathrm{~S}$ ribosomal subunit of prokaryotic ribosomes. The clinically relevant antibacterial spectrum of tobramycin includes staphylococci, notably Staphylococcus aureus and Staphylococcus epidermidis. All streptococci, including Streptococcus pneumoniaeand enterococci are resistant. Tobramycin is active against many aerobic and facultative gram-negative bacilli, including Enterobacteriaceae, Pseudomonas aeruginosa (P. aeruginosa), and Acinetobacter spp. Although active in vitro, tobramycin is not used clinically for infections caused by Haemophilus spp. and Legionella spp. due to low intracellular concentration. Tobramycin is also not the preferred aminoglycoside for mycobacterial infections or infections caused by Yersinia spp. and Francisella spp. As with all aminoglycosides, tobramycin requires aerobic metabolism to exert its effect, thus it does not have activity against anaerobic bacteria.

When combined with various other antimicrobacterial agents, tobramycin has demonstrated in vitro synergy against a variety of gram-positive and gram-negative organisms, including enterococci, $S$. aureus, S. epidermidis, enterobacteriaceae, $P$. aeruginosa, and corynebacteria JK. Gentamicin or streptomycin remains the preferred aminoglycoside for synergism against enterococcal infections, and the therapeutic relevance of in vitro synergy of tobramycin against gramnegative organisms is difficult to evaluate in the clinical setting.

Current infectious diseases guidelines do not list aminoglycosides as first choice agents due to their toxicity potential and the availability of equally efficacious regimens [2,3]. Hence, the clinical role of aminoglycosides is limited to the urinary tract, combination therapy, or treating documented resistant infections when no better alternatives exist. Tobramycin may be used for infections originating from most body sites, except for the central nervous system, due to poor penetration.

Tobramycin is still being used in treating urinary tract infections due to its excellent urinary concentration. However, tobramycin's main role is in combination with a beta-lactam agent against Gram-negative bacterial pathogens. In the empiric setting, the aim of combination therapy is to provide a broad-spectrum empiric regimen that is likely to include at least one agent active against the suspected etiologic bacterial pathogen. This practice is specifically endorsed and detailed in the latest healthcare pneumonia guideline [4], and is particularly important in patients who are critically ill or suspected of having infections caused by multi-drug resistance organisms. In certain infections with documented susceptibilities, tobramycin may be added as part of a combination regimen due to its rapid bactericidal effect and when the infection is difficult to eradicate. This is true in the setting of cystic fibrosis and Pseudomonas endocarditis. Lastly, with the insurgence of multidrug resistant gram-negatives, clinicians may be compelled to use tobramycin when no other agents remain susceptible, often at the expense of increased toxicity.

Clinical response rates of $90 \%$ or greater are achieved when peak aminoglycoside concentrations are 8 to 10 times higher than the MIC of the infecting organism. Like amikacin and gentamicin, side effects such as renal toxicity, ototoxicity, vestibular toxicity, and neuromuscular blockade have made tobramycin the less than ideal agent. However, the increasing incidence of MDR organisms has seen a resurgence of utility for tobramycin and other aminoglycoside agents.

Two specific areas where tobramycin continue to be beneficial are discussed.

\section{Cystic Fibrosis}

Tobramycin plays a major role in the management of lung disease for both adult and pediatric populations with Cystic Fibrosis (CF) because of its activity against $P$. aeruginosa.

$P$. aeruginosa is the most prevalent colonizing organism in the airway of CF patients. Up to $30 \%$ of patients aged $<2$ years can have positive $P$. aeruginosa cultures, and increases to $80 \%$ in patients aged $>18$ years [5]. This organism enters the lower airways presumably by inhalation from the environment and may transiently infect the airways of 10 to $50 \%$ of CF patients. Early P. aeruginosa colonization usually evolves to chronic persistence of this organism. These strains undergo a phenotypic change, which is characterized by the production of large amounts of an extracellular mucoid polysaccharide known as alginate. Motile P. Aeruginosa contained in the thickened mucus coat overlying the CF airway epithelium undergo adaptation to nonmotile, anaerobically respiring mucoid variants growing as a biofilm [6].

*Corresponding author: Milagros $P$ Reyes, Division of Infectious Diseases Harper University Hospital,3990 John R, Detroit, MI 48201, USA, Tel: 313-7457105; Fax: 313-993-0302; E-mail: mreyes@med.wayne.edu

Received December 13, 2013; Accepted February 19, 2014; Published February 28, 2014

Citation: Reyes MP, Zhao JJ, Buensalido JAL (2014) Current Perspectives: Therapeutic Uses of Tobramycin. J Pharmacovigilance 2: 123. doi:10.4172/23296887.1000123

Copyright: (๑) 2014 Reyes MP, et al. This is an open-access article distributed under the terms of the Creative Commons Attribution License, which permits unrestricted use, distribution, and reproduction in any medium, provided the original author and source are credited. 
Parallel to this, in the early stage of pseudomonal infection in CF, the isolates are usually susceptible to all antipseudomonal beta-lactams, quinolones, and aminoglycosides. With increasing antimicrobial pressure and the emergence of chronic mucoid strains, antibiotic resistance increases as well.

Lung damage secondary to chronic infection is the major cause of morbidity and mortality in individuals with CF. Persistent or recurrent infections result in a prolonged inflammatory response, which is believed to cause respiratory tissue injury leadingto progressive deterioration of lung function and respiratory failure, which is the leading cause of death in CF patients.

Repeated and intensive antibiotic therapy is required to maintain lung function and quality of life and to reduce exacerbations in infected patients. Antibiotic therapy aimed at eradicating P. aeruginosa, the major bacterial pathogen in CF after early lung infections, and improved regimens to treat chronic $P$. aeruginosa infection, have played a major role in increasing the median survival of CF during the last decade. In 1969, CF patients in industrialized countries had a mean survival of 14 years. This has improved to 40 years in some centers by 2010 [7].

\section{Intravenous Tobramycin}

Intravenous tobramycin is indicated in the management of Acute Pulmonary Exacerbations (APE) of CF, to control chronic infection, and to eradicate $P$. aeruginosa after recent acquisition. The Cystic Fibrosis Pulmonary Guidelines recommend treating an APE with an aminoglycoside (IV) and an anti-pseudomonal agent for synergy and to reduce development of resistance [8]. Tobramycin is the most commonly utilized aminoglycoside among $98 \%$ of CFF-accredited care pediatric centers in the US; amikacin, second [9]. The routine use of gentamicin in the treatment of APE is not recommended due to a lack of studies showing efficacy and evidence indicating an increased risk of nephrotoxicity compared to tobramycin. The drugs and duration recommended for combination therapy with an aminoglycoside (usually tobramycin) are anti-pseudomonalpenicillins (piperacillin) or cephalosporins (ceftazidime, cefepime), monobactam (aztreonam) or carbapenems (imipenem, meropenem) for 2-3 weeks [10,11].

Once-Daily Dosing (ODD) of tobramycin (in combination with ceftazidime) has been shown to be as effective as a thrice daily regimen and less nephrotoxic for APE [12]. Administration of a single large dose per day achieves a higher post dose serum concentration and allows time for aminoglycoside levels to fall prior to administration of the next dose. This is currently the preferred mode of administration of tobramycin for APE. Studies have supported a tobramycin ODD of 10 $\mathrm{mg} / \mathrm{kg} /$ day, targeting tobramycin serum $\mathrm{C}_{\max }$ and $\mathrm{C}_{\min }$ concentrations of 20-40 and $<1 \mathrm{mg} / \mathrm{L}$ with a targeted mean AUC of $60-120 \mathrm{mg} \mathrm{hr} / \mathrm{L}$ [13].

Recently, two publications have shown that an empiric tobramycin regimen of $10 \mathrm{mg} / \mathrm{kg} /$ day does not achieve desired peak serum concentrations of $20-30 \mathrm{mg} / \mathrm{L}$. Only $42 \%$ of patients achieved the desired serum concentrations in one study of 14 children and 11 adults, although all patients showed clinical improvement.The authors recommend that these desired tobramycin concentrations could be achieved with an empiric dosage of $12 \mathrm{mg} / \mathrm{kg} /$ day and that a prospective evaluation of this regimen with individualized TDM be performed [14]. In the second study, a re-evaluation of tobramycin treatment guidelines for the CF population is suggested for adults with APE with a tobramycin MIC of 2 or $4 \mathrm{mg} / \mathrm{L}$ for P. aeruginosa. MICs of tobramycin for $P$. aeruginosa are rising and the authors suggest that a higher dosing strategy of $15-20 \mathrm{mg} / \mathrm{kg} / \mathrm{day}$ would increase the probability of clinical response and reduce the probability for emergence of resistance for these patients [15].

Tobramycin dosages used in the CF population are higher than that used in the non-CF population. Studies have shown that CF patients tend to have a larger volume of distribution and greater renal and total clearance for aminoglycosides $[9,16]$. This translates to higher doses of tobramycin in the CF patients in order to achieve similar serum concentrations to those without CF. Despite these higher dosages, the rapid clearance in a CF patient may still result in a prolonged drug free interval where the time below the organism MIC exceeds that of the tobramycin post antibiotic effect. This would be detrimental both microbiologically and clinically. Hence, regimens in the CF population need to be individualized based on Therapeutic Drug Monitoring (TDM).

The recurrent nature of respiratory exacerbations of $\mathrm{CF}$ and increasing lifespan of patients with CF lead to a considerable lifetime exposure to aminoglycosides (tobramycin). Short term complications such as acute kidney injury and vestibular toxicity, and longer term cumulative toxicities such as hearing loss/balance problems and chronic kidney disease have been well documented [17,18]. TDM of intravenous aminoglycosides (tobramycin) is essential for maximizing efficacy and minimizing toxicity.

Currently empiric dosages of tobramycin can be derived using weight based dosing or PK equations which rely on estimated patient parameters such as volume of distribution and creatinine clearance. Ideally the MIC of the Pseudomonas isolate from the most recent culture should be used to empirically determine the desired peak tobramycin concentration. TDM in the CF population is similar to the non-CF population in that serum concentrations are measured to subsequently derive at patient specific PK parameters, which are then used to adjust a dosage regimen specific to the patient. There are also nomograms available for tobramycin dosage adjustment, such as the Hartford nomogram [19]. However these nomograms are based on a fixed $\mathrm{mg} / \mathrm{kg}$ dose requires assumptions that patient kinetic parameters are comparable, and were validated in non-CF populations $[19,20]$ These limitations must be taken into consideration when nomograms are applied to CF patients.

Despite improved results with the current combined IV antibiotic regimen using tobramycin, there is still a need for further studies to determine the optimal tobramycin dose, dosing interval and pharmacokinetic/pharmacodynamics (PK/PD) targets for the treatment of APE. The optimum method for TDM also remains to be determined.

\section{Nebulized Tobramycin}

Inhaled administration of tobramycin in patients with $\mathrm{CF}$ who have chronic $P$. aeruginosa infection enables the delivery of sufficient concentrations of antibiotic directly to the site of infection and allows a bactericidal effect with reduced systemic absorption and toxicity [21]. The use of Tobramycin Inhaled Solution (TIS) in patients with CF has been associated with improvement in lung function, decreased frequency of hospitalizations, and decreased endobronchial burden of $P$. aeruginosa [22]. TIS is generally prescribed for use in alternate monthly cycles consisting of $300 \mathrm{mg}$ of TIS twice daily for 28 days, followed by 28 days off therapy.

The 2013 Cystic Fibrosis Pulmonary Guidelines continue to strongly recommend the chronic use of inhaled tobramycin to reduce exacerbations in individuals with mild, moderate and severe 
CF, 6 years of age and older and P. aeruginosa persistently present in cultures of the airways [23]. The chronic use of inhaled tobramycin is also recommended to improve lung function and quality of life in CF patients 6 years of age and older, with moderate to severe disease and $P$. aeruginosa persistently present in cultures of the airways. A new recommendation added in 2013 supports the chronic use of inhaled aztreonam for individuals with CF, 6 years or older, with mild, moderate and severe disease, and $P$. aeruginosa persistently present in cultures of the airways. The CF Foundation concludes that the evidence is insufficient to recommend for or against the chronic use of other inhaled antibiotics such as ceftazidime, colistin or gentamicin to improve lung function, quality of life or reduce exacerbations

The absorption of inhaled tobramycin following the standard dose is highly variable. Mean (and median) peak concentration is approximately $1 \mathrm{mg} / \mathrm{L}$ with a reported range of $0.18-3.62 \mathrm{mg} / \mathrm{L}$ using the Pari LC' Plus nebulizer [24]. Advanced nebulization systems such as the Parie Flow have higher delivery efficiency. Serum tobramycin levels were $>1 \mathrm{mg} / \mathrm{L}$ in 3 children; however urine NAG ( $\mathrm{N}$-acetyl-beta$\mathrm{D}$-glucosaminidase) levels were increased in 5 children suggesting early toxicity. One patient even experienced high frequency hearing loss [25]. TDM and routine audiology screening should be performed in patients receiving nebulized tobramycin alone via the eFlow and when co-prescribed with intravenous (IV) tobramycin.

Despite the ability to nebulize aminoglycosides such as tobramycin, there is no evidence to support their use during APE in addition to IV anti-pseudomonal beta-lactam antibiotics. However, a recent study investigated the effect of inhaled tobramycin on serum concentrations in 20 adult CF patients admitted to the hospital for APE and concomitantly treated with parenteral tobramycin [26]. TIS were given 5-9 hours after the IV dose. A significant increase in serum tobramycin trough level $(>/=1 \mathrm{ug} / \mathrm{mL})$ was seen in 9 patients; 14 patients had a reduced calculated elimination rate constant after TIS administration. The authors suggest that timing of the inhaled dose should be considered when interpreting PK measures of IV tobramycin dosing. This study did not offer insights into efficacy or toxicity.

\section{Inhaled Tobramycin Formulations}

Tobramycin Inhalation Solution (TIS) Was the First PreservativeFree Aerosol Antibiotic Approved for CF. anEarlier Preparation Which Contained phenol as preservative $\left(\mathrm{Nebicina}^{\mathrm{R}}\right)$ lost favor because of unpleasant taste [27]. Tobramycin is also available in another nebulized formulation (Bramitob ${ }^{\mathrm{R}}$, Chiesi). The total dose is the same as for TIS, but it is more concentrated $(75 \mathrm{mg} / \mathrm{ml}$ versus $60 \mathrm{mg} / \mathrm{ml}$ ) to decrease nebulization time. Currently available tobramycin inhalation solutions have the $\mathrm{pH}$ and salinity adjusted specifically for administration by a compressed air driven reusable nebulizer.

Tobramycin has also been developed as a dry powder formulation (TOBI $^{\mathrm{R}}$; Podhaler ${ }^{\mathrm{R}}$; TIP ${ }^{\mathrm{TM}}$, Novartis) for the management of chronic $P$. aeruginosa infection in CF patients. Tobramycin Inhalation Powder (TIP) capsules are formed of low density porous particles (Pulmo Sphere ${ }^{\mathrm{TM}}$ ) which show improved flow and dispersion by inhalation via the passive T-326 dry powder inhaler $\left(\right.$ Podhaler $\left.^{\mathrm{R}}\right)$. Two controlled studies showed that the Podhaler formulation displayed similar tolerability and efficacy to TIS and significantly improved FEV1 compared with placebo [28]. Cough was the most frequently reported adverse reaction related to the dry powder in both clinical studies.

TIP has been shown to have significantly faster treatment times and achieves higher patient satisfaction than TIS. No external power source is required to deliver an efficacious tobramycin dose in minutes compared to TIS. The recommended dose is $112 \mathrm{mg}$ (four 28 mg capsules) inhaled twice daily in alternating cycles of 28 days on treatment followed by 28 days off treatment [29].

\section{Pseudomonas endocarditis}

Tobramycin continues to play a major role in the treatment of endocarditis due to P. aeruginosa. Pseudomonas endocarditis (PsE) is an uncommon disease. The risk factors for developing PsE are parenteral drug use, implanted prosthetic cardiac valves and endovascular devices. In a review by the International Collaboration on Endocarditis Prospective Cohort Study (ICE-PCS), Morpeth et al. [30] found that 49 of 2761 (1.8\%) patients had endocarditis due to non-HACEK gramnegative bacilli. The predominant risk factor for non-HACEK gramnegative bacillus endocarditis was health care contact (57\%) whereas injection drug use was rare (4\%). Twenty-nine of the 49 patients $(59 \%)$ had prosthetic endocarditis which was defined as endocarditis involving a prosthetic heart valve or implanted cardiovascular device such as a permanent cardiac pacemaker, cardioverter defibrillator, or aortic stent. In-hospital mortality rate was high (24\%) despite high rates of cardiac surgery (51\%). Mortality rate among the 11 patients with pseudomonas endocarditis in this series was $36 \%(4 / 11)$.

In Detroit, injection drug use continues to be the most common predisposing factor for the development of PsE.

P. aeruginosa accounted for $10-13.5 \%$ of endocarditis cases in Injection Drug Users (IDUs) between 1969 and 1984 [31,32]. Between 1969 and 1975, among 37 cases of PsE seen at the DMC, tricuspid valve was involved in 70\% (26/37) and 22\% (8/37) had left-sided disease [33]. Three patients $(3 / 37 ; 8 \%)$ had both left- and right-sided involvement. Medical cure rate for tricuspid disease was 44\% (11/25); however outcome improved to $80 \%(20 / 25)$ with carbenicillin plus highdose aminoglycoside ( $8 \mathrm{mg} / \mathrm{kg} /$ day in 3 divided doses) and tricuspid valvulectomy without valve replacement. Medical cure rate for leftsided disease was 33\% (3/9). All 3 patients with left-sided disease who had surgery died. Overall mortality of PsE was $32 \%$.

A review of 15 IDUs with left-sided PsEat the Detroit Medical Center (DMC)between 1980 and 1984 showed that medical therapy alone with an anti-pseudomonal penicillin andhigh-dose tobramycin $(8 \mathrm{mg} / \mathrm{kg} /$ day $)$ in 3 divided doses resulted in poor outcomes \{MR 6/7; $85.7 \%\}$ [34]. Outcome appeared to improve to $62.5 \%(5 / 8)$ with surgery. However, overall mortality of left-sided PsE remained high $(9 / 15 ; 60 \%)$ with a 5 year follow-up. The outcome of left-sided PsE is worse than right-sided disease because serious complications associated with left-sided PsE are frequent and include neurologic squealed, ring and annular abscesses, congestive heart failure and splenic abscesses [34].

In the most recent series between 2006-2008 at the DMC, a new antimicrobial regimen was used consisting of a combination of cefepime, $6 \mathrm{~g} /$ day, in 3 divided doses, plus ODD of tobramycin, $8 \mathrm{mg} / \mathrm{kg}$ per day, for 6 weeks [35]. Peak tobramycin serum levels of $18-22 \mathrm{ug} / \mathrm{ml}$ and trough levels of $<1 \mathrm{ug} / \mathrm{ml}$ were aimed for. Actual peak tobramycin levels achieved ranged from $15-24 \mathrm{ug} / \mathrm{ml}$. The overall medical cure rate for both left-sided and right-sided PsE was 80\% (4/5).The patient who did not survive had aortic valve involvement and could not undergo surgery because of a large embolic cerebral infarct. All 5 patients who required surgery survived $(5 / 5 ; 100 \%)$. Overall mortality rate was $10 \%$ $(1 / 10)$ compared to $32 \%(11 / 34)$ in the previous collective study from 1969-1975 where gentamicin or tobramycin was administered in a thrice daily intermittent regimen with an antipseudomonal penicillin.

Although this was a small series there was a trend toward better 
recovery with surgery for left-sided PsE. Two patients with pure left-sided prosthetic valve PsE underwent successful repeat valve replacements. A third patient with persistent pseudomonas bacteremia and congestive heart failure underwent successful replacement of a native aortic valve. Tricuspid valvulectomy with valve replacement was successful in 3 patients. Earlier, tricuspid valvulectomy without valve replacement used to be advocated as the surgical procedure of choice for antibiotic refractory PsE of the tricuspid valve [36]. However, severe and permanent impairment of right ventricular function in a relatively young population is an undesirable long-term complication of this procedure.

Nephrotoxicity occurred in 2/10 (20\%) patients in the 2006-2008 study. There was no systematic monitoring for auditory or vestibular toxicity in this study. One patientshowed high frequency hearing loss consistent with aminoglycoside toxicity. In a previous study of the renal and auditory toxicity of 15 patients with PsE who received 17 courses of high dose, prolonged therapy with gentamicin or tobramycin, renal dysfunction and auditory toxicity occurred in 5/8 (63\%) and $4 / 9$ (44\%), respectively, of gentamicin treated patients and in $3 / 7$ (43\%) and 2/8 (25\%), respectively, of tobramycin treated patients [37]. Vestibular toxicity occurred in one patient in each group. Gentamicin or tobramycin was given in 3 divided doses of $8 \mathrm{mg} / \mathrm{kg} / \mathrm{day}$. Peak serum levels of $12-15 \mathrm{ug} / \mathrm{ml}$ were achieved with a trough of $<2 \mathrm{ug} / \mathrm{ml}$. A high incidence of nephrotoxicity and auditory toxicity was seen with intermittent dosing of either aminoglycoside.

Once daily dosing of tobramycin utilizes a higher dose of the antibiotic but at a longer interval ( 24 hours) compared with traditional dosing strategies. This dosing strategy decreases the likelihood of toxicity due to slower uptake into the proximal renal tubular epithelial cell, allows a longer period of time for drug elimination, thus decreases the probability of drug accumulation, and optimizes the post antibiotic effect [38]. It also allows higher doses to be employed which can better achieve target concentrations for effective killing, especially in settings of increased organism MIC. Unfortunately, the benefit of once daily dosing of tobramycin is limited by renal function. In patients with renal insufficiency, in order to achieve the same serum peak concentration to attain efficacy, the dosing interval will need to be prolonged to more than 24 hours to attain undetectable trough concentrations at the end of the dosing interval, thus increasing the risk of cumulative drug exposure and toxicity. In the setting of multidrug resistant infections, this increased risk of toxicity may be unavoidable if the aminoglycoside remains the only therapeutic option.

With tobramycin TDM, ideally a peak concentration should be determined to evaluate efficacy based on peak to MIC ratio, and a trough concentration should be determined to evaluate clearance, thus toxicity. In clinical practice, it is often difficult to obtain levels this precisely; hence two serum concentrations are advocated from the same dosing interval which can be used to calculate the patient's PK parameters including peak and trough concentrations, and to tailor subsequent dosing. Typically, 18 hour serum concentrations should be less than 1 $\mathrm{ug} / \mathrm{ml}$ in order to achieve undetectable levels at the end of a 24 hours dosing interval. Again, in the setting of multidrug resistant organisms and renal insufficiency, in order to maintain the same efficacy, a high dose regimen may still be needed which can result in sustained serum concentration above $1 \mathrm{ug} / \mathrm{ml}$ at the end of 24 hours. This necessitates a dosing interval longer than 24 hours and an increased risk for toxicity. If possible, audiometry should be performed at baseline and patients should be assessed weekly once on prolonged tobramycin therapy. Signs of vestibular toxicity should also be monitored clinically. Renal function should be checked at baseline and at least two or three times a week, and more frequently if renal function is unstable. Finally, efforts should be made to avoid other concomitant nephrotoxic agents while the patient is on tobramycin.

The current antimicrobial regimen for PsE at the DMC consists of a combination of cefepime, $6 \mathrm{~g} / \mathrm{d}$, in 3 divided doses, plus ODD of tobramycin, $8 \mathrm{mg} / \mathrm{kg}$ per day. For cefepime-resistant pseudomonas, imipenem, 4-6 g/d, or meropenem, $6 \mathrm{~g} / \mathrm{d}$ plus ODD of tobramycin has been successful [35]. For right-sided disease refractory to medical therapy, surgical intervention is recommended if pseudomonas bacteremia persists for 2 weeks on appropriate antimicrobial therapy or if bacteremia recurs after a 6-week course of treatment. Tricuspid valvulectomy with valve replacement plus combined antipseudomonal regimen may be the optimal therapy for refractory right-sided PsE. For left-sided disease, surgery is recommended if blood cultures remain positive for 7 days on appropriate antimicrobial therapy or if pseudomonas bacteremia recurs after completion of a 6-week course of the combined regimen [35].

In summary, once daily dosing of tobramycin (in combination with a beta lactam agent) using $10 \mathrm{mg} / \mathrm{kg} /$ day appears to be more effective and less toxic than intermittent thrice daily dosing for APEs in cystic fibrosis patients. Once daily dosing with tobramycin at a dose of $8 \mathrm{mg} /$ $\mathrm{kg} /$ day (in combination with cefepime or imipenem or meropenem) appears to be at least as effective and less toxic than thrice daily dosing for the treatment of pseudomonas endocarditis.

Additional studies are needed to define serum target levels and efficacy with once daily high dose regimens for tobramycin (in combination with a beta lactam agent) for the treatment of APEs in cystic fibrosis as well as for the treatment of pseudomonas endocarditis. These studies should include the optimum method for TDM, baseline and regular monitoring of auditory and vestibular function.

\section{References}

1. Koch KF, Rhoades JA (1970) Structure of nebramycin factor 6, a new aminoglycosidic antibiotic. Antimicrob Agents Chemother (Bethesda) 10: 309313.

2. Solomkin JS, Mazuski JE, Bradley JS, Rodvold KA, Goldstein EJ, et al. (2010) Diagnosis and management of complicated intra-abdominal infection in adults and children: guidelines by the Surgical Infection Society and the Infectious Diseases Society of America. Clin Infect Dis 50:133-164.

3. Freifeld AG, Bow EJ Sepkowitz KA, Boeckh MJ, Ito Jl et al (2011) Clinica practice guideline for the use of antimicrobial agents in neutropenic patients with cancer: 2010 update by the infectious diseases society of america. Clin Infect Dis 52: e56-93.

4. American Thoracic Society; Infectious Diseases Society of America (2005) Guidelines for the management of adults with hospital-acquired, ventilatorassociated, and healthcare-associated pneumonia. Am J Respir Crit Care Med 171: $388-416$.

5. Cantón R, Cobos N, de Gracia J, Baquero F, Honorato J, et al. (2005) Antimicrobial therapy for pulmonary pathogenic colonisation and infection by Pseudomonas aeruginosa in cystic fibrosis patients. Clin Microbiol Infect 11 : 690-703.

6. Donaldson SH, Wolfgang MC, Gilligan PH, et al. (2010) Cystic Fibrosis. Mandell, Douglas, and Bennett's principles and practice of infectious diseases. Mandell GL, Bennett JE, Dolin R Editors, Volume 1, Churchill Livingstone Elsevier, Philadelphia, USA, 947-955

7. Döring G, Flume P, Heijerman H, Elborn JS (2012) Treatment of lung infection in patients with cystic fibrosis: current and future strategies. J Cyst Fibros 11 : 461-479.

8. Flume PA, Mogayzel PJ Jr, Robinson KA, Goss CH, Rosenblatt RL, et al. (2009) Cystic fibrosis pulmonary guidelines: treatment of pulmonary exacerbations. Am J Respir Crit Care Med 180: 802-808. 
Citation: Reyes MP, Zhao JJ, Buensalido JAL (2014) Current Perspectives: Therapeutic Uses of Tobramycin. JPharmacovigilance 2: 123. doi:10.4172/23296887.1000123

Page 5 of 5

9. Young DC, Zobell JT, Stockmann C, Waters CD, Ampofo K, et al. (2013) Optimization of anti-pseudomonal antibiotics for cystic fibrosis pulmonary exacerbations: V. Aminoglycosides. Pediatr Pulmonol 48: 1047-1061.

10. Döring G, Conway SP, Heijerman HG, Hodson ME, Høiby N, et al. (2000) Antibiotic therapy against Pseudomonas aeruginosa in cystic fibrosis: a European consensus. Eur Respir J 16: 749-767.

11. Smith AL, Doershuk C, Goldmann D, Gore E, Hilman B, et al. (1999) Comparison of a beta-lactam alone versus beta-lactam and an aminoglycoside for pulmonary exacerbation in cystic fibrosis. J Pediatr 134: 413-421.

12. Smyth A, Tan KH, Hyman-Taylor P, Mulheran M, Lewis S, et al. (2005) Once versus three-times daily regimens of tobramycin treatment for pulmonary exacerbations of cystic fibrosis--the TOPIC study: a randomised controlled trial. Lancet 365: 573-578.

13. Burkhardt O, Lehmann C, Madabushi R, Kumar V, Derendorf H, et al. (2006) Once-daily tobramycin in cystic fibrosis: better for clinical outcome than thricedaily tobramycin but more resistance development? J Antimicrob Chemother 58: 822-829.

14. Vandenbussche HL, Homnick DN (2012) Evaluation of serum concentrations achieved with an empiric once-daily tobramycin dosage regimen in children and adults with cystic fibrosis. J Pediatr Pharmacol Ther 17: 67-77.

15. Butterfield JM, Lodise TP, Beegle S, Rosen J, Farkas J, et al. (2013) Pharmacokinetics and pharmacodynamics of once-daily administration of intravenous tobramycin in adult patients with cystic fibrosis hospitalized for an acute pulmonary exacerbation. Antimicrob Agents Chemother 57: 5175-5177.

16. Touw DJ, Vinks AA, Neef C (1997) Pharmacokinetic modelling of intravenous tobramycin in adolescent and adult patients with cystic fibrosis using the nonparametric expectation maximization (NPEM) algorithm. Pharm World Sc 19: $142-151$

17. Braccini G, Lori I, Neri AS, Festini F, Pela I, et al. (2009) Parenteral administration of tobramycin for pulmonary exacerbations in cystic fibrosis patients: toxicity, serum levels and efficacy. J Chemother 21: 101-104.

18. Prayle A, Smyth AR (2010) Aminoglycoside use in cystic fibrosis: therapeutic strategies and toxicity. Curr Opin Pulm Med 16: 604-610.

19. Nicolau DP, Freeman CD, Belliveau PP, Nightingale CH, Ross JW, et al. (1995) Experience with a once-daily aminoglycoside program administered to 2,184 adult patients. Antimicrob Agents Chemother 39: 650-655

20. Urban AW, Craig WA (1997) Daily dosage of aminoglycosides. Curr Clin Top Infect Dis 17: 236-255.

21. Lenoir G, Antypkin YG, Miano A, Moretti P, Zanda M, et al. (2007) Efficacy, safety, and local pharmacokinetics of highly concentrated nebulized tobramycin in patients with cystic fibrosis colonized with Pseudomonas aeruginosa. Paediatr Drugs 9 Suppl 1: 11-20.

22. Ramsey BW, Dorkin HL, Eisenberg JD, Gibson RL, Harwood IR, et al. (1993) Efficacy of aerosolized tobramycin in patients with cystic fibrosis. N Engl J Med 328: $1740-1746$
23. Mogayzel PJ Jr, Naureckas ET, Robinson KA, Mueller G, Hadjiliadis D, et al. (2013) Cystic fibrosis pulmonary guidelines. Chronic medications for maintenance of lung health. Am J Respir Crit Care Med 187: 680-689.

24. Geller DE, Pitlick WH, Nardella PA, Tracewell WG, Ramsey BW (2002) Pharmacokinetics and bioavailability of aerosolized tobramycin in cystic fibrosis. Chest 122: 219-226.

25. Guy EL, Bosomworth M, Denton M, Conway SP, Brownlee KG, et al. (2010) Serum tobramycin levels following delivery of tobramycin (Tobi) via eFlow advanced nebuliser in children with cystic fibrosis. J Cyst Fibros 9: 292-295.

26. Stenbit AE, Bullington WM, Heh JL, Flume PA (2013) Timing of inhaled tobramycin affects assessment of intravenous tobramycin pharmacokinetic monitoring. J Cyst Fibros 12: 403-406.

27. Nikolaizik WH, Trociewicz K, Ratjen F (2002) Bronchial reactions to the inhalation of high-dose tobramycin in cystic fibrosis. Eur Respir J 20: 122-126.

28. Konstan MW, Flume PA, Kappler M, Chiron R, Higgins M, et al. (2011) Safety, efficacy and convenience of tobramycin inhalation powder in cystic fibrosis patients: The EAGER trial. J Cyst Fibros 10: 54-61.

29. Vandevanter DR, Geller DE (2011) Tobramycin administered by the TOBI(®) Podhaler $(\circledR)$ for persons with cystic fibrosis: a review. Med Devices (Auckl) 4 179-188.

30. Morpeth S, Murdoch D, Cabell CH, Karchmer AW, Pappas P, et al. (2007) NonHACEK gram-negative bacillus endocarditis. Ann Intern Med 147: 829-835.

31. El-Khatib MR, Wilson FM, Lerner AM (1976) Characteristics of bacterial endocarditis in heroin addicts in Detroit. Am J Med Sci 271: 197-201.

32. Levine DP, Crane LR, Zervos MJ (1986) Bacteremia in narcotic addicts at the Detroit Medical Center. II. Infectious endocarditis: a prospective comparative study. Rev Infect Dis 8: 374-396.

33. Reyes MP, Lerner AM (1983) Current problems in the treatment of infective endocarditis due to Pseudomonas aeruginosa. Rev Infect Dis 5: 314-321.

34. Komshian SV, Tablan OC, Palutke W, Reyes MP (1990) Characteristics of left-sided endocarditis due to Pseudomonas aeruginosa in the Detroit Medical Center. Rev Infect Dis 12: 693-702.

35. Reyes MP, Ali A, Mendes RE, Biedenbach DJ (2009) Resurgence of Pseudomonas endocarditis in Detroit, 2006-2008. Medicine (Baltimore) 88 : 294-301.

36. Arbulu A, Holmes RJ, Asfaw I (1991) Tricuspid valvulectomy withou replacement. Twenty years' experience. J Thorac Cardiovasc Surg 102: 917922.

37. Tablan OC, Reyes MP, Rintelmann WF, Lerner AM (1984) Renal and auditory toxicity of high-dose, prolonged therapy with gentamicin and tobramycin in pseudomonas endocarditis. J Infect Dis 149: 257-263.

38. Drusano GL, Louie A (2011) Optimization of aminoglycoside therapy. Antimicrob Agents Chemother 55: 2528-2531. 